\title{
EFEITO RESIDUAL DE QUATRO APLICAÇÕES ANUAIS DE LODO DE ESGOTO E VINHAÇA NA QUALIDADE TECNOLÓGICA DA CANA-DE-AÇÚCAR
}

\author{
CARAMO CÓ JÚNIOR ${ }^{1}$, MARCOS O. MARQUES ${ }^{2}$, LUIZ C. TASSO JÚNIOR ${ }^{3}$
}

\begin{abstract}
RESUMO: O principal objetivo deste trabalho foi avaliar os efeitos residuais, por quatro anos seguidos, de diferentes tipos de resíduos, aplicados de formas e em doses distintas, sobre a qualidade tecnológica da cana-de-açúcar de quinto corte (cultivar SP 81-3250). Os tratamentos testados são combinações de dois tipos de resíduos (lodo de esgoto; vinhaça; lodo de esgoto + vinhaça) com dois modos de aplicação (ao lado da linha de cultivo e em área total) e com duas doses (100 e 200\%), e um tratamento-testemunha. O delineamento experimental foi em blocos casualizados, com três repetições, totalizando 39 parcelas. As variáveis analisadas foram: percentagem de sólidos solúveis no caldo extraído (Brix \% C.E.), percentagem aparente de sacarose no caldo extraído (Pol \% C.E.), percentagem da fibra na cana (Fibra \% cana), pureza (\%), percentagem aparente de sacarose na cana (Pol \% cana), percentagem de açúcares redutores na cana (AR \% cana), percentagem de açúcares redutores totais na cana (ART \% cana) e açúcares totais recuperáveis (ATR). Os fatores de variação testados (tipo de resíduo, modo de aplicação e doses) não promoveram alterações na qualidade e não comprometeram a valorização da cana-de-açúcar.
\end{abstract}

PALAVRAS-CHAVE: resíduos, uso agrícola, Saccharum spp.

\section{SUGARCANE TECHNOLOGYCAL PARAMETERS AFFECTED BY SEWAGE SLUDGE AN VINASSE ADDED IN SOIL FOR FOUR CONSECUTIVE YEARS}

\begin{abstract}
The main objective of this research was to evaluate the residual effect of different residues applied during four years in distinct application modes and rates, on the technological quality of the $5^{\text {th }}$ ratoon of sugar cane (cultivar SP 81-3250). The treatments were from combination of two residues (sewage sludge, vinasse, sewage sludge + vinasse) applied according two different ways (close to the sugar cane line and spread in the whole area), in two rates (100 and $200 \%$ ) besides a control treatment. The experimental design was randomized blocks with thirteen treatments and three replications, resulting 39 experimental plots. The following parameters were analyzed: percentage of soluble solids in juice (Brix \% JC), apparent percentage of sucrose in juice (Pol \% JC), percentage of fiber in sugar cane (Fiber \% Cane), purity (\%), apparent percentage of sucrose in sugar cane (Pol \% Cane), percentage of reducing sugar in sugar cane (RS \% Cane), percentage of total reducing sugar in sugar cane (TRS \% Cane), recoverable total sugar content in sugar cane (RTRS). The evaluated factors (type of residues, application way and rates) did not cause alterations on the technological parameters and sugar cane commercial value.
\end{abstract}

KEYWORDS: residues, agricultural use, Saccharum spp.

\footnotetext{
${ }^{1}$ Eng $^{0}$ Agrônomo, Doutorando no Programa de Pós-Graduação em Agronomia (Produção Vegetal), UNESP - Câmpus de Jaboticabal, Via de Acesso Prof. Paulo Donato Castellane, km 5, Jaboticabal - SP, Fone/fax: (0XX16) 3209 2675, ccojunior@yahoo.com.br

${ }^{2}$ Professor Adjunto, Departamento de Tecnologia, UNESP - Câmpus de Jaboticabal - SP, Fone/fax: (0XX16) 3209 2675, omir@fcav.unesp.br

${ }^{3}$ Eng $^{\mathrm{o}}$ Agrônomo, Doutor em Agronomia, Departamento de Tecnologia, UNESP - Câmpus de Jaboticabal - SP, Fone/fax: (0XX16) 3209 2675, 1ctasso@yahoo.com.br

Recebido pelo Conselho Editorial em: 10-4-2007
}

Aprovado pelo Conselho Editorial em: 12-12-2007 


\section{INTRODUÇÃO}

O crescimento populacional e a conseqüente demanda por bens de consumo, assim como o aumento do desenvolvimento industrial geram, principalmente nas regiões metropolitanas, águas residuárias e resíduos sólidos em quantidades vultosas (FRANCO, 2003).

O lodo de esgoto gerado em Estações de Tratamento de Esgotos (ETEs) pode ser definido como material sólido, constituído basicamente de matéria orgânica, elementos nutrientes de plantas e metais pesados, além de microrganismos patogênicos ou não (MARQUES, 1990).

Com o objetivo de destacar o potencial de utilização para fins produtivos e minimizar o estigma de resíduo, esse material, uma vez higienizado, estabilizado e seco, passou a ser denominado biossólido (MARQUES et al., 2002). Sua composição depende do material que lhe deu origem, mas, em média, apresenta de 60 a 80 dag kg${ }^{-1}$ de umidade, sendo cerca de 30 a $40 \mathrm{dag} \mathrm{kg}^{-}$ ${ }^{1}$ de matéria orgânica na fração sólida, além de elementos minerais nutrientes de plantas (macro e micronutrientes), podendo, ainda, conter metais pesados, compostos orgânicos complexos oriundos de produtos domiciliares e/ou industriais e patógenos humanos (NOBILE, 2002).

Entre as possibilidades de destinação final do lodo de esgoto, citam-se a incineração, o lançamento nos oceanos (emissários submarinos) e a deposição em aterros sanitários, mas nenhuma reúne aspectos suficientes para torná-las mais interessantes do que o seu emprego como fertilizante ou condicionador de solos agrícolas. O potencial para tanto decorre da presença de matéria orgânica e elementos minerais (MARQUES et al., 1997).

Entretanto, a presença de patógenos humanos, metais pesados e baixos teores de potássio em sua composição é problema que deve ser equacionado para viabilizar a referida prática sem comprometer a produção das culturas (COSTA et al., 2001).

A agroindústria sucroalcooleira, da mesma forma, gera resíduos provenientes do processamento industrial da cana-de-açúcar, em quantidades que se correlacionam diretamente com seu porte. Destacam-se, entre os resíduos gerados, a água de lavagem da cana, as cinzas de caldeira, a torta de filtro e a vinhaça, que, apesar de apresentarem elevado potencial poluidor, não causam preocupação quando previamente tratados e utilizados novamente no processo (água de lavagem de cana) ou quando utilizados como fertilizantes ou condicionadores de solos em geral, no cultivo da própria cana. Essa opção é de grande interesse, pois, além de dar destino aos resíduos, torna-os úteis na medida em que, por sua decomposição no solo, interferem positivamente em seus atributos, especialmente em termos de fertilidade.

A vinhaça é o subproduto da fabricação do álcool, sendo composta, em sua maioria, de água

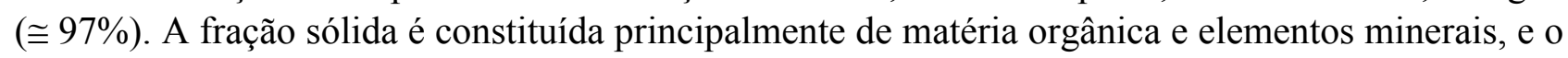
K representa cerca de $20 \%$ dos elementos presentes e constitui o elemento limitante para a definição da dose a ser aplicada nos solos (MARQUES, 2006).

O Brasil tem capacidade instalada para a produção de álcool da ordem de 16 bilhões de litros ao ano (UNICA, 2007), e o volume produzido de vinhaça é de 12 a 15 vezes maior. No total, a cana-de-açúcar ocupa cerca de três milhões de hectares no Estado de São Paulo (FRONZALIA, 2007). A maior parte dessa área $(75-80 \%)$ pode perfeitamente receber vinhaça por meio de fertirrigação.

A disponibilidade de nutrientes oriundos de resíduos orgânicos é dependente da taxa de degradação da matéria orgânica no solo, sendo esse processo lento e incompleto em um mesmo ano agrícola (FERREIRA et al., 1999). Elevados teores de matéria orgânica no solo resultam em atraso no processo de maturação da cana-de-açúcar (MARQUES et al., 2001).

Dessa forma, neste trabalho, foram analisados os efeitos residuais de quatro aplicações anuais de lodo de esgoto sanitário, como fonte de nitrogênio, e de quatro aplicações vinhaça, como fonte de potássio, em comparação a cinco aplicações anuais de fertilizantes minerais, em variáveis agroindustriais da cana-soca (quinto corte). 


\section{MATERIAL E MÉTODOS}

O experimento foi iniciado em 7 de abril de 2000 e conduzido no município de Pontal - SP, em área de propriedade da Destilaria Santa Inês, localizada na Rodovia Sertãozinho-Pontal (SP322 ), com as seguintes coordenadas geográficas: latitude: $21^{\circ} 01^{\prime} 26^{\prime}$ 'S e longitude: $48^{\circ} 02^{\prime} 18^{\prime}$ 'W, em Latossolo Vermelho eutroférico, cuja análise química resultou nos seguintes valores: $\mathrm{pH}$ $\left(\mathrm{CaCl}_{2}\right)=5,0 ; \mathrm{M} . \mathrm{O}\left(\mathrm{g} \mathrm{dm}^{-3}\right)=25 ; \mathrm{P}$ resina $\left(\mathrm{mg} \mathrm{dm}{ }^{-3}\right)=20 ; \mathrm{K}\left(\mathrm{mmol}_{\mathrm{c}} \mathrm{dm}^{-3}\right)=1,2 ; \mathrm{Ca}\left(\mathrm{mmol}_{\mathrm{c}} \mathrm{dm}^{-3}\right)$ $=20 ; \mathrm{Mg}\left(\mathrm{mmol}_{\mathrm{c}} \mathrm{dm}^{-3}\right)=6 \mathrm{e} \mathrm{H}+\mathrm{Al}\left(\mathrm{mmol}_{\mathrm{c}} \mathrm{dm}^{-3}\right)=28$.

O lodo de esgoto foi empregado como fonte de nitrogênio, e a vinhaça, como fonte de potássio. Dessa forma, a dose de $100 \%$ foi calculada de forma que a quantidade aplicada de lodo de esgoto contivesse a quantidade de nitrogênio recomendada para a cultura, de acordo com RAIJ et al. (1997). Para a vinhaça, o mesmo procedimento foi adotado em relação ao potássio. A dose de $200 \%$ constituiu-se no dobro da quantidade referente à dose de $100 \%$.

Os tratamentos testados foram repetidos anualmente, nos quatro primeiros ciclos da cultura. $\mathrm{O}$ delineamento experimental adotado foi em blocos ao acaso, constituídos de 13 tratamentos, resultantes da combinação de três tipos de resíduos (lodo de esgoto, vinhaça e lodo de esgoto + vinhaça) com as duas doses (100 e 200\%) e dois modos de aplicação (no sulco de plantio - canaplanta ou ao lado da linha - soqueiras e espalhado em área total), mais um tratamento-testemunha, com três repetições, totalizando 39 parcelas. Para a análise estatística, os resultados foram organizados segundo esquema Fatorial $3 \times 2 \times 2$, mais um tratamento-testemunha, sendo empregado o programa "Statistical Analysis System" (SAS INSTITUTE, 1989).

Nas parcelas que receberam fertilizantes minerais (tratamento-testemunha), as quantidades aplicadas foram calculadas de acordo com as recomendações de RAIJ et al. (1997).

No quinto ciclo da cultura, as parcelas correspondentes ao tratamento-testemunha receberam fertilizantes. Nas demais, nada foi aplicado com o intuito de verificar possíveis efeitos residuais das aplicações realizadas nos quatro primeiros anos.

As parcelas experimentais, num total de 39, constituíram-se de cinco linhas de cana com comprimento de $10 \mathrm{~m}$ e espaçamento de $1,50 \mathrm{~m}$ entre linhas, totalizando $75 \mathrm{~m}^{2}$. Por ocasião da amostragem, procedeu-se ao descarte de $1 \mathrm{~m}$ de cada uma das extremidades das parcelas e da primeira e da quinta linha de cana, coletando-se as amostras nas três linhas centrais.

O lodo de esgoto, digerido, seco ao ar, foi obtido junto à SABESP (Companhia de Saneamento Básico do Estado de São Paulo) - Estação de Tratamento de Esgotos de Franca - SP.

A vinhaça empregada foi proveniente da destilação de vinho de caldo na Destilaria Santa Inês Ltda., localizada no município de Pontal - SP. Tanto o lodo de esgoto como a vinhaça foram amostrados e, nas amostras, procedeu-se à determinação de $\mathrm{N}, \mathrm{P}, \mathrm{K}, \mathrm{Ca}, \mathrm{Mg}, \mathrm{Mn}, \mathrm{Fe}, \mathrm{Cu}$ e $\mathrm{Zn}$, de acordo com a metodologia proposta por MALAVOLTA et al. (1989). Os resultados obtidos nos quatro primeiros anos de cultivo são apresentados na Tabela 1.

A cultivar utilizada (SP 81-3250) caracteriza-se por apresentar período útil de industrialização médio, de ótima brotação de soqueira, alto teor de fibra e de sacarose (COPERSUCAR, 1995). De acordo com NUNES JÚNIOR et al. (2004), essa cultivar foi a mais cultivada no Estado de São Paulo, na safra de 2003/2004, representando $14,23 \%$ do total da cana colhida. Considerando a região centro-sul, a mesma também foi a mais cultivada $(11,38 \%)$.

Em 25-8-2005 (quinto ano), foram coletados dez colmos escolhidos ao acaso, de cada parcela experimental, os quais foram enfeixados, etiquetados e encaminhados ao laboratório de pagamento de cana da Destilaria Santa Inês, onde foram desintegrados, homogeneizados e analisados de acordo com a metodologia do CONSECANA (2005). 
TABELA 1. Composição química do lodo de esgoto e da vinhaça durante os quatros primeiros anos de aplicação. Chemical composition of sewage sludge and vinasse during the first four crop years.

\begin{tabular}{|c|c|c|c|c|c|c|c|c|c|}
\hline Resíduo/ano & $\mathrm{N}$ & $\mathrm{P}$ & $\mathrm{K}$ & $\mathrm{Ca}$ & $\mathrm{Mg}$ & $\mathrm{Mn}$ & $\mathrm{Fe}$ & $\mathrm{Cu}$ & $\mathrm{Zn}$ \\
\hline Lodo de esgoto & \multicolumn{5}{|c|}{------------------- g kg } & \multicolumn{4}{|c|}{-------------- $\mathrm{mg} \mathrm{kg}^{-1}$------------- } \\
\hline $1^{\mathrm{o}}$ & 79,5 & 10,6 & 0,63 & -- & -- & 400 & 26.391 & 225 & $1.000,0$ \\
\hline $2^{-}$ & 52,0 & 3,4 & 1,96 & 4,3 & 0,6 & 98 & 29.350 & 380 & 658,0 \\
\hline $3^{\mathrm{o}}$ & 39,8 & 6,8 & 0,50 & 0,5 & 0,0 & 723 & 60.900 & 110,7 & 416,7 \\
\hline $4^{\mathrm{o}}$ & 52,0 & 3,4 & 0,60 & 4,3 & 0,6 & 676 & 61.033 & 117 & 393,3 \\
\hline Vinhaça & \multicolumn{5}{|c|}{ 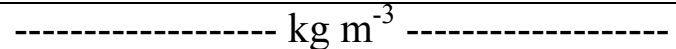 } & \multicolumn{4}{|c|}{ 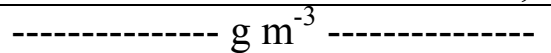 } \\
\hline $1^{\underline{0}}$ & 0,39 & 0,06 & 4,08 & 2,6 & 0,2 & 4,5 & 59,89 & 0,17 & 0,82 \\
\hline $2^{\underline{o}}$ & 0,30 & 0,09 & 3,83 & 2,7 & 0,2 & 4,2 & 63,42 & 0,12 & 0,76 \\
\hline $3^{\mathrm{o}}$ & 0,36 & 0,34 & 1,05 & 1,1 & 0,3 & 7,4 & 69,75 & 1,12 & 2,65 \\
\hline $4^{\underline{O}}$ & 0,40 & 0,01 & 0,85 & 1,2 & 0,4 & 8,6 & 75,25 & 2,05 & 4,47 \\
\hline
\end{tabular}

Foram determinadas as seguintes variáveis agroindustriais: percentagem de sólidos solúveis no caldo extraído (Brix \% CE), percentagem aparente de sacarose do caldo extraído (Pol \% CE), percentagem de açúcares redutores no caldo (AR \% CE) e percentagem da fibra na cana (Fibra \% cana). De posse dos resultados, procedeu-se aos cálculos da pureza aparente, percentagem de sacarose na cana (Pol \% cana), percentagem de açúcares redutores na cana (AR \% cana), percentagem de açúcares redutores totais na cana (ART \% cana) e açúcar total recuperável (ATR) expresso em $\mathrm{kg} \mathrm{t}^{-1}$.

\section{RESULTADOS E DISCUSSÃO}

Os resultados das análises de Brix e Pol do caldo (Tabela 2) serviram de base para o cálculo da pureza. Por meio dessas avaliações, constata-se que os fatores de variação testados (tipo de resíduo, formas de aplicação e doses) não influenciaram na qualidade do caldo, nem sofreram alterações suficientes para torná-las diferentes dos resultados encontrados nas parcelas que receberam fertilizantes minerais (Testemunha). De modo geral, os valores médios de Brix oscilaram entre 21,36 e $21,66 \%$. Neste trabalho, os valores encontrados estão acima dos limites mencionados na bibliografia como os mínimos necessários para que a cana apresente condições de ser amostrada para a realização de uma análise tecnológica detalhada, com o objetivo de caracterizar o seu grau de maturação, que, de acordo com MARQUES et al. (2001), é de $18 \%$.

Os valores médios de Pol situam-se entre 20,07 e 20,45\%. A não-ocorrência de efeitos dos fatores de variação sobre a referida variável contraria as expectativas, na medida em que vão de encontro às afirmações de STUPIELLO et al. (1977) e SOBRAL et al. (1981), os quais atribuem ao aumento da matéria orgânica e à adição de fertilizantes nos solos, o atraso no processo de maturação da cana.

Quanto aos valores de pureza, esses situaram-se entre 93,96 e 94,45\%, indicando que a matéria-prima, no momento do corte, apresentava mais do que o valor mínimo de pureza necessário para a sua industrialização, que é de $80 \%$ no início e de $85 \%$ no transcorrer da safra (MARQUES et al., 2001).

Dessa forma, fica descartada a possibilidade de os resíduos terem contribuído para menor pureza do caldo, decorrente, por exemplo, do aumento nos teores de açúcares redutores que, se ocorressem sem o comprometimento dos teores de sacarose, seria interessante na medida em que contribuiriam para maior taxa de recuperação de sacarose na forma cristalizada, durante a fase de cozimento do xarope, por diminuir o coeficiente de solubilidade da sacarose (CHEN \& CHOU, 1993). 
TABELA 2. Variáveis agroindustriais da cana-de-açúcar ( $5^{-}$corte) em função do tipo de resíduo aplicado, forma de aplicação e doses empregadas, nos quatro primeiros anos de cultivo. Valores médios e resumo da análise estatística. Agroindustrial parameters of sugar cane $\left(5^{\circ}\right.$ ratoon-cane) according to residue type, application mode and rates, in the first four crop years. Average values and statistical analysis summary.

\begin{tabular}{|c|c|c|c|c|c|c|c|c|}
\hline $\begin{array}{c}\text { Fatores } \\
\text { Resíduo (R) }\end{array}$ & --- \% CE --- & $\begin{array}{r}\text { Pol } \\
\text { CE --- }\end{array}$ & $\begin{array}{c}\text { Pureza } \\
(\%)\end{array}$ & Fibra & $\begin{array}{l}\text { Pol } \\
--\% ~ C ~\end{array}$ & $\begin{array}{c}\text { AR } \\
\text { ana ---- }\end{array}$ & ART & $\begin{array}{c}\text { ATR } \\
\left(\mathrm{kg} \mathrm{t}^{-1}\right)\end{array}$ \\
\hline Lodo de esgoto (L) & 21,47 & 20,21 & 94,13 & 11,63 & 17,23 & 0,07 & 18,21 & 160,18 \\
\hline Vinhaça (V) & 21,51 & 20,25 & 94,10 & 12,13 & 17,10 & 0,10 & 18,10 & 159,25 \\
\hline $\mathrm{L}+\mathrm{V}$ & 21,55 & 20,34 & 94,38 & 12,11 & 17,19 & 0,07 & 18,16 & 159,98 \\
\hline Teste F & $0,04 \mathrm{~ns}$ & $0,09 \mathrm{~ns}$ & $0,23 \mathrm{~ns}$ & $1,13 \mathrm{~ns}$ & $0,12 \mathrm{~ns}$ & $1,09 \mathrm{~ns}$ & $0,08 \mathrm{~ns}$ & $0,09 \mathrm{~ns}$ \\
\hline DMS $(5 \%)$ & 0,77 & 0,84 & 1,13 & 0,94 & 0,64 & 0,05 & 0,66 & 5,87 \\
\hline \multicolumn{9}{|l|}{ Iodo de aplicação (M) } \\
\hline Lin & 1,58 & 20,35 & 94,31 & 12,1 & 17,19 & 0,07 & 18,17 & 159,9 \\
\hline Área & 21,44 & 20,18 & 94,1 & 11,8 & 17,15 & 0,09 & 18,14 & 159,71 \\
\hline Test & $0,03 \mathrm{~ns}$ & $0,38 \mathrm{~ns}$ & 0,331 & $1,04 \mathrm{~ns}$ & $0,03 \mathrm{~ns}$ & $0,71 \mathrm{~ns}$ & $0,02 \mathrm{~ns}$ & $0,01 \mathrm{~ns}$ \\
\hline DMS & 0,52 & 0,57 & 0,76 & 0,64 & 0,43 & 0,03 & 0,45 & 3,96 \\
\hline \multicolumn{9}{|l|}{ Dose ( D ) } \\
\hline $100 \%$ & 21,66 & 20,45 & 94,45 & 12,07 & 17,3 & 0,07 & 18,28 & 160,94 \\
\hline $20 c$ & 21,36 & 20,07 & 93,96 & 11,83 & 17,05 & 0,09 & 18,03 & 158,67 \\
\hline Teste F & $1,36 \mathrm{~ns}$ & $1,91 \mathrm{~ns}$ & $1,17 \mathrm{~ns}$ & $0,61 \mathrm{~ns}$ & $1,43 \mathrm{~ns}$ & $1,09 \mathrm{~ns}$ & $1,33 \mathrm{~ns}$ & $1,40 \mathrm{~ns}$ \\
\hline DMS $(5 \%)$ & 0,52 & 0,57 & 0,76 & 0,64 & 0,43 & 0,03 & 0,45 & 3,96 \\
\hline \multicolumn{9}{|l|}{ Interações } \\
\hline $\mathrm{R} \times \mathrm{M}$ & $0,89 \mathrm{~ns}$ & $0,47 \mathrm{~ns}$ & 0,23 & 1,07 & $0,27 \mathrm{~ns}$ & $2,06 \mathrm{~ns}$ & $0,33 \mathrm{~ns}$ & $0,30 \mathrm{~ns}$ \\
\hline $\mathrm{R} \times \mathrm{D}$ & $1,39 \mathrm{~ns}$ & $1,06 \mathrm{~ns}$ & 0,51 & $0,08 \mathrm{~ns}$ & $1,47 \mathrm{~ns}$ & $0,27 \mathrm{~ns}$ & $1,45 \mathrm{~ns}$ & $1,49 \mathrm{~ns}$ \\
\hline$M \times D$ & $0,81 \mathrm{~ns}$ & $0,92 \mathrm{~ns}$ & $0,42 \mathrm{~ns}$ & $0,36 \mathrm{n}$ & $1,75 \mathrm{~ns}$ & $2,95 \mathrm{~ns}$ & $1,49 \mathrm{~ns}$ & $1,65 \mathrm{~ns}$ \\
\hline $\mathrm{R} \times \mathrm{M} \times \mathrm{D}$ & $0,16 \mathrm{~ns}$ & $0,16 \mathrm{~ns}$ & $0,04 \mathrm{~ns}$ & $0,03 n$ & $0,26 \mathrm{~ns}$ & $2,31 \mathrm{~ns}$ & $0,18 \mathrm{~ns}$ & $0,18 \mathrm{~ns}$ \\
\hline \multicolumn{9}{|l|}{ Médias } \\
\hline Teste & ,53 & 0,11 &, 38 & 11,45 & 17,18 & 0,13 & 18,21 & 160,26 \\
\hline Fat & 21,51 & 20,26 & 94,20 & 11,95 & 17,17 & 0.08 & 18,16 & 159,80 \\
\hline Tes & $0,00 \mathrm{r}$ & $0,10 \mathrm{r}$ & $1,54 \mathrm{r}$ & $0,82 \mathrm{n}$ & $0,00 \mathrm{~ns}$ & $2,43 \mathrm{~ns}$ & $0,02 \mathrm{~ns}$ & $0,02 \mathrm{~ns}$ \\
\hline C. V. $(\%)$ & 3,52 & 4,07 & 1,17 & 7,75 & 3,68 & 59,04 & 3,56 & 3,6 \\
\hline
\end{tabular}

ns - não-significativo; DMS - diferença mínima significativa; C.V. - coeficiente de variação.

Os valores de Fibra \% Cana, juntamente com as variáveis determinadas no caldo, serviram de base para os cálculos das respectivas variáveis relacionadas à cana.

Os teores de fibra na cana variaram de 11,63 a 12,13\%. Valores entre 11 e $16 \%$ podem ser considerados normais (MEADE, 1963); BAIKOW (1982) estabelece o valor de $15 \%$ como valor médio para cana industrial, ou seja, cana disposta na esteira da usina. Nesse mesmo experimento, em ciclos anteriores ( $1^{\underline{0}}$ ao $4^{\underline{0}}$ corte), os teores de fibra oscilaram entre $9 \%$ e $15 \%$ (FRANCO, 2003; CAMILOTTI et al., 2006).

Verifica-se que os fatores de variação não promoveram alterações nas variáveis relativas à cana, e essas, por sua vez, não apresentaram diferenças daquelas obtidas nas parcelas que receberam fertilizantes minerais (Testemunha).

Os valores de Pol \% Cana variaram de 17,05 a 17,30\%, indicando que, no momento do corte, a cana se apresentava madura, de acordo com DEUBER (1988), o qual afirma que o teor mínimo para essa condição é de $13 \%$.

Os teores de AR \% Cana ocorreram em níveis entre 0,07 e $0,10 \%$, sendo esse indicativo de que a cana pode ser considerada apta à industrialização, levando-se em conta que, no início de safra, esses valores podem ser de até $1,5 \%$, não devendo superar $1 \%$ no transcorrer da safra (MARQUES et al., 2001). 
Os resultados de ART \% Cana foram calculados a partir dos valores de Pol \% Cana e AR \% cana. De forma geral, os valores situaram-se na faixa entre 18,03 e 18,28\%. Essas informações, somadas aos valores AR \% Cana, permitiram o cálculo da quantidade de Açúcar Total Recuperável, que expressa o potencial da indústria em recuperar o açúcar contido na cana, na forma de açúcar cristal ou de etanol. Neste trabalho, os valores de ATR variam de 158,67 a 160,94 $\mathrm{kg} \mathrm{t}^{-1}$. Esses resultados são semelhantes aos encontrados por TASSO JÚNIOR (2007) que, estudando dez cultivares de período útil de industrialização médio, em dois municípios da região centro-norte do Estado de São Paulo, encontrou, no momento do corte, valores entre 137 e $166 \mathrm{~kg} \mathrm{t}^{-1}$. Especificamente para a cultivar estudada neste trabalho, o autor destaca valores entre 150 e $166 \mathrm{~kg} \mathrm{t}^{-1}$.

Do ponto de vista ambiental, a aplicação de resíduos no solo, em área total, contempla o efeito de diluição do mesmo, sugerindo essa forma de aplicação. Por outro lado, quando se considera o uso de resíduos na agricultura, o aproveitamento dos nutrientes e os efeitos benéficos que eventualmente tais produtos possam promover no solo são de interesse primordial. Assim, partindose desses princípios, pode-se afirmar que o uso localizado apresenta vantagens que estão relacionadas à eficiência agrícola do resíduo como fertilizante.

Na Figura 1, apresenta-se a análise de correlação linear entre os valores de ART \% Cana e ATR $\left(\mathrm{kg} \mathrm{t}^{-1}\right)$ das parcelas cujos tratamentos receberam os resíduos testados.

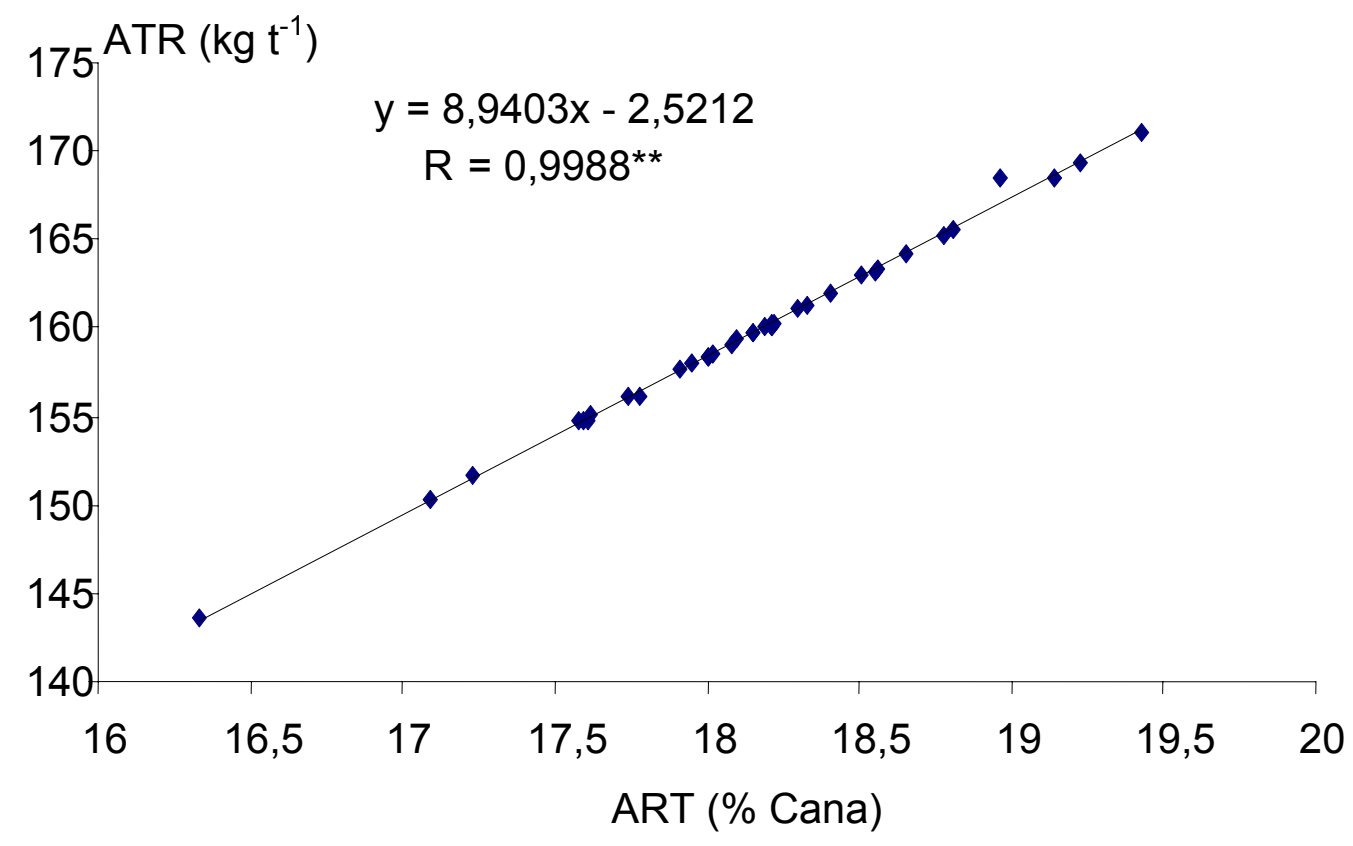

FIGURA 1. Reta obtida por análise de correlação linear entre ART \% Cana e ATR $\left(\mathrm{kg} \mathrm{t}^{-1}\right)$. Linear correlation obtained from Total Reducing Sugar \% Cane and Recoverable Total Sugar $\left(\mathrm{kg} \mathrm{t}^{-1}\right)$.

Os dados apresentados na Figura 1 demonstram que se estabelece correlação positiva entre os valores de ART \% Cana e ATR. Além disso, o elevado valor do coeficiente de correlação $\left(\mathrm{R}=0,9988^{* *}\right)$ demonstra que os fatores de variação estudados não influenciaram de formas distintas essas duas variáveis.

Com a equação da reta obtida, possibilita-se o cálculo da ATR da cana fertilizada com resíduos, doses e modos de aplicação estudados. Como se pode verificar na Tabela 2, o tratamentotestemunha proporcionou valores médios de ART e ATR de 18,21\% e 160,26 $\mathrm{kg} \mathrm{t}^{-1}$, respectivamente. 
Substituindo o valor de ART médio do tratamento-testemunha na equação da Figura 1, tem-se o valor de ATR de 160,28 $\mathrm{kg} \mathrm{t}^{-1}$. Isso permite a inferência de que os resíduos empregados, nas formas de aplicações e doses estudadas, não promoveram alterações nas variáveis tecnológicas da matéria-prima a ponto de influenciar na ATR, e, por conseguinte, na valorização da mesma.

\section{CONCLUSÕES}

Os efeitos residuais de aplicações de lodo de esgoto e vinhaça, em suas diferentes combinações, doses e formas de aplicação, não influenciaram na qualidade tecnológica da cana-deaçúcar (soqueira de quinto corte).

O uso dos resíduos, em comparação ao da fertilização mineral, não alterou a qualidade da matéria-prima, destacando o expressivo potencial dos mesmos para essa função.

\section{REFERÊNCIAS}

BAIKOW, V.E. Manufacture and refining of raw cane sugar. $2^{\text {nd }}$ ed. Amsterdam: Elsevier, 1982. $588 \mathrm{p}$.

CAMILOTTI, F.; ANDRIOLI, I.; MARQUES, M.O.; SILVA, A.R.; TASSO JUNIOR, L.C.; NOBILE, F.O.; NOGUEIRA, G.A.; PRATI, F. Produtividade e qualidade agroindustrial da canade-açúcar cultivada com lodo de esgoto, vinhaça e adubos minerais. STAB, Piracicaba, v.24, n.3, p.32-5, 2006.

CHEN, J.C.P.; CHOU, C. Cane sugar handbook. 12 ${ }^{\text {th }}$ ed. New York: John Wiley, 1993. 284 p.

CONSECANA. CONSELHO DOS PRODUTORES DE CANA-DE-AÇÚCAR, AÇÚCAR E ÁlCOOL DO ESTADO DE SÃO PAULO. Manual de instruções. 4. ed. Piracicaba, 2005. 115 p.

COPERSUCAR. COOPERATIVA DOS PRODUTORES DE CANA, AÇÚCAR E ÁLCOOL DO ESTADO DE SÃO PAULO. $5^{\underline{a}}$ geração de variedades. Piracicaba, 1995. p.16-23 (Boletim Técnico).

COSTA, A.N.; COSTA, A.F.S.; MARQUES, M.O.; SANTANA, R.C. Estudo de caso - utilização de lodo de estações de tratamento de esgoto (ETEs) na cultura de mamoeiro no norte de Estado de Espírito Santo. In: ANDRIOLI, C.V. (Coord.) Resíduos sólidos do saneamento: processamento, reciclagem e disposição final. Rio de Janeiro: Rima/ABES, 2001. p.189-214.

DEUBER, R. Maturação da cana-de-açúcar na região Sudeste do Brasil. In: SEMINÁRIO DE TECNOLOGIA DA COPERSUCAR, 1988, Piracicaba. Anais... Piracicaba: Centro de Tecnologia Copersucar, 1988. p.33-40.

FRANCO, A. cana-de-açúcar cultivada em solo adubado com lodo de esgoto e vinhaça: nitrogênio no sistema solo planta, produtividade e características tecnológicas. 2003. 90 f. Dissertação (Mestrado em Produção Vegetal) - Faculdade de Ciências Agrárias e Veterinárias, Universidade Estadual Paulista, Jaboticabal, 2003.

FRONZALIA, T. Cana-de-açúcar: expansão alarmante. Instituto de Economia Agrícola, São Paulo. Disponível em: http://www.iea.sp.gov.br/out/verTexto.php?codTexto=8905. Acesso em: 2 abr. 2007.

FERREIRA, A.C.; ANDREOLI, C.V.; JURGENSEN, D. II. Destino final do lodo. In: ANDREOLI, C.V.; LARA, A.I.; ILHENFELD, R.G.K. Uso e manejo do lodo de esgoto na agricultura. Rio de Janeiro: PROSAB, 1999. p.26-28.

MALAVOLTA, E.; VITTI, G.C.; OLIVEIRA, S.A. Avaliação do estado nutricional das plantas: princípios e aplicações. Piracicaba: Associação Brasileira para a Pesquisa da Potassa e do Fosfato, 1989. p.135-39. 
MARQUES, M.O. Aspectos técnicos e legais da produção, transporte e aplicação de vinhaça. In: SEGATO, S.V.; PINTO, A.S.; JENDIROBA, E.; NÓBREGA, J.C.M. (Org.). Atualização em produção de cana-de-açúcar. Piracicaba: Editoral 2006. p.369-375.

MARQUES, M.O. Efeitos da aplicação do lodo de esgoto na produtividade e qualidade da canade-açúcar. 1990. 164 f. Tese (Doutorado em Solos e Nutrição de Plantas) - Escola Superior de Agricultura "Luiz de Queiroz”, Universidade de São Paulo, Piracicaba, 1990.

MARQUES, M.O.; MARQUES, T.A.; TASSO JÚNIOR, L.C. Tecnologia do açúcar: produção e industrialização da cana-de-açúcar. Jaboticabal: FUNEP, 2001. 166 p.

MARQUES, M.O.; MELO, W.J.; CAMILOTTI, F.; TASSO JÚNIOR, L.L.; NOBILE, F.O. Sugarcane parameters in biosolid and vinasse added soil. In: FAZ CANO, A.; ORTIZ SILLA, R.; MERMUT, A.R. Sustainable use and management of soils in and semiarid regions. Catagena: Quaderna Editorial, 2002. v.2, p.549-50.

MARQUES, M.O.; MELO, W.J.; BERLLINGIERI, P.A.; MARRETO, G.H.; KANESIRO, M.A.; MARQUES, T.A.; CHELLI, R.A.; LEITE, S.A.S. Residual effect of sewage sludge on the fertility of a soil copped with sugar-cane. In: DROZ, J.; GONET, S.S.; SENESI, N.; WEBER, J. The role of humu substances in the ecosystems and in envinonmental protection. Wroclaw: PTSH, 1997, p.88388.

NOBILE, F.O. Variáveis agroindustriais da cana-de-açúcar cultivada em solo acrescido com lodo de esgoto e vinhaça. 2002. 66 f. Monografia. (Trabalho de Graduação em Agronomia) - Faculdade de Ciências Agrárias e Veterinárias, Universidade Estadual Paulista, Jaboticabal, 2002.

NUNES JÚNIOR, D.; PINTO, A.R.S.A.; TRENTO FILHO, E.; ELIAS, A.I. Indicadores agrícolas do setor sucroalcooleiro: safra 2003/04. Ribeirão Preto: IDEA, 2004. 111 p.

RAIJ, B. van; CANTARELA, H.; QUAGGIO, J.A.; FURLANI, A.M.C. Recomendações de adubação e calagem para o Estado de São Paulo. Campinas: Instituto Agronômico, 1997. p 23739. (Boletim Técnico, 100).

SAS INSTITUTE. SAS language and procedures: Usage. Version 6. Cary, 1989. 78 p.

SOBRAL, A.F.; CORDEIRO, D.D.; SANTOS, M.A.C. Efeito da aplicação de vinhaça em socarias de cana-de-açúcar. Brasil Açucareiro, Rio de Janeiro, v.98, n.5, p.52-8, 1981.

STUPIELLO, J.P.; PEXE, C.A.; MONTEIRO, H.; SILVA, L.H. Efeitos da aplicação de vinhaça como fertilizante na qualidade da cana-de-açúcar. Brasil Açucareiro, Rio de Janeiro, v.90, n.3, p.41-50, 1977.

TASSO JÚNIOR, L.C. Caracterização agrotecnológica de cultivares de cana-de-açúcar (Saccharum spp.) na região centro-norte do Estado de São Paulo. 2007. 167 f. Tese (Doutorado em Produção Vegetal) - Faculdade de Ciências Agrárias e Veterinárias, Universidade Estadual Paulista, Jaboticabal, 2007.

ÚNICA. UNIÃO DA INDÚSTRIA DE CANA-DE-AÇÚCAR. São Paulo. Produção de álcool do Brasil, abril 2007. Disponível em: http://www.portalunica.com.br/portalunica/index.php?Secao=referência\&SubSecao=estatísticas\&S ubSacao=produção\%20Brasil\&id=\%20and\%id=9. Acesso em: 2 abr. 2007. 